\title{
Distributed Temperature Sensing System Based on Brillouin Scattering Effect Using a Single-Photon Detector
}

\author{
Liwen Sheng $\left(\mathbb{D},{ }^{1,2,3,4}\right.$ Jisong Yan, ${ }^{1}$ Ligong Li, ${ }^{1}$ Ming Yuan ${ }^{1},{ }^{1}$ Shuai Zhou, ${ }^{1}$ Rui Xu, \\ Jiaqing Liu, ${ }^{1,4}$ Fushun Nian $\mathbb{D}^{1},{ }^{2,4}$ Long $\mathrm{Li}^{3},{ }^{3}$ and Zhiming Liu ${ }^{1}$ \\ ${ }^{1}$ Ceyear Technologies Co., Ltd., Qingdao 266555, China \\ ${ }^{2}$ The 41st Institute of CETC, Qingdao 266555, China \\ ${ }^{3}$ Xidian University, Xi'an 710071, China \\ ${ }^{4}$ Science and Technology on Electronic Test \& Measurement Laboratory, Qingdao 266555, China \\ Correspondence should be addressed to Ming Yuan; ymfjysfj@163.com and Fushun Nian; fushunnian_ceyear@163.com
}

Received 10 February 2021; Revised 9 March 2021; Accepted 16 April 2021; Published 22 April 2021

Academic Editor: Zhenxu Bai

Copyright (C) 2021 Liwen Sheng et al. This is an open access article distributed under the Creative Commons Attribution License, which permits unrestricted use, distribution, and reproduction in any medium, provided the original work is properly cited.

Utilizing a single-photon detector, a novel direct-detection optical-fiber sensor for distributed measurement of temperature based on spontaneous Brillouin scattering is proposed and demonstrated experimentally. In our scheme, the ratio of the backscattered Rayleigh signal and the backscattered Brillouin anti-Stokes is adopted to retrieve the monitored temperature information along the optical fiber. Taking advantage of the high sensitivity of the single-photon detector, our proposed system achieves a dynamic range of $20 \mathrm{~dB}$ without any optical amplification. The obtainable dynamic range corresponds to a sensing distance of $120 \mathrm{~km}$ with a measured temperature error of $0.96^{\circ} \mathrm{C}$. Furthermore, the proof-of-concept experiment demonstrates $1.2 \mathrm{~m}$ spatial resolution over $4.2 \mathrm{~km}$ sensing link with $1.24^{\circ} \mathrm{C}$ temperature error. Considering the performance we achieved now, and the increasing improvement of the fabrication technology of sing-photon detector, the photon-counting distributed Brillouin sensor is opening a door in the field of optical-fiber sensors.

\section{Introduction}

As one of the strongest nonlinear optical phenomena, Brillouin scattering has been discovered and widely studied for several decades. It has attracted intensive interests in time/frequency domain beam shaping [1-5], the high gain Brillouin enhancement of weak signals [6-8], and distributed temperature sensing systems in recent years $[9,10]$. For the fiber temperature sensors case, the Brillouin-based distributed fiberoptic temperature sensors are an attractive alternative to traditional point sensors, because they are not only adopting a standard single mode fiber (SMF) as the common fiberoptic sensing elements to replace potentially thousands of individual sensors, but also inheriting features from classical fiberoptic sensors, such as low cost, durability, smallness in size, and stability. This makes Brillouin-based distributed temperature measuring systems especially attractive in the civil engineering areas, such as the structural health monitoring (SHM) of large facilities, long-distance temperature measurements for pipelines, and others.

These Brillouin-based distributed fiberoptic sensors can mainly be split into two classes: the Brillouin reflectometer (optical time domain-BOTDR) [11-13] and Brillouin analyzer (optical time domain-BOTDA) [14-16]. Among them, the first type (BOTDR) is considered as one of the most promising techniques that can retrieve distributed ambient temperature information by single-ended fiber under test (FUT) architecture, large dynamic range, and random accessibility. It typically adopts a modulated probe pulse to interrogate the FUT and demodulate position information from the time-of-flight of the backscattered Brillouin photons from the sensing fiber. Temperature acts as the most common monitoring parameter in BOTDR systems, as this is the quantity to which the FUT is inherently sensitive. In general, the change in temperature conditions of the sensing optical fiber induces variations in the acoustic 
properties of the Brillouin medium and, by consequence, can be demodulated by examination of the Brillouin frequency shift (BFS) monitored. Most of BOTDR sensing devices developed so far are based on the temperature and strain dependence of the BFS [9]. Therefore, the coherent heterodyne detection and frequency sweep [17] are required in these presented Brillouin-based sensing systems to obtain the Brillouin gain spectrum (BGS) with high signal noise ratio (SNR). Then, the BFS, which is dependent on both applied temperature and strain along the sensing fiber, can be achieved by Lorentz curve fitting the BGS. As a result, the sensing system configuration would be rather complicated, and the process of frequency tuning to monitor the BGS would be very time-consuming.

Remarkably, in Brillouin-based distributed temperature systems (DTS), namely, BOTDR DTS systems, the ambient temperature information can also be measured from the intensity ratio of the backscattered Rayleigh component and the spontaneous Brillouin scattering (SpBS) component (i.e., Stokes component and anti-Stokes component) which was first reported experimentally by Schroeder et al. [18]. However, the separation with high rejection ratio of the SpBS signal from the Rayleigh scattering (RS) signal problem is a key challenge. It is evident that the residual RS component in SpBS signal would evolve a strong background noise when the Brillouin component is measured, which gives rise to a significant temperature error. To resolve the key problem effectively, many scholars have done a lot of researches to accomplish the intensity measurement of RS and SpBS signal, such as fiber Bragg grating (FBG) notch filter [19], Fabry-Perot scanning interferometer [20], and Mach-Zehnder interferometer [21]. But the typical frequency shift value between Stokes/anti-Stokes component and Rayleigh is about $10.85 \mathrm{GHz}(\sim 0.0868 \mathrm{~nm})$ in common silica optical fibers. Therefore, as mentioned above all, these proposed methods are not able to obtain relatively pure Brillouin signal. In addition, to enlarge the temperature sensing distance limited by the power of the weak signal and photon-detector's (PD) sensitivity, some groups employed optical preamplification method to solve this sensing range issue [22]. Unfortunately, owing to the involvement of optically pumped amplifiers, the BOTDR DTS sensor would give rise to a large ASE-ASE beat noise which easily deteriorates the sensing performance. On the other hand, the more sensible approach for obtaining long measuring distance without amplifying the laser pulse would be to enhance the sensitivity of the receiver. At present, utilizing singlephoton detectors (SPD) with a high sensitivity of about $-100 \mathrm{dBm}$ instead of linear PD in conventional OTDR systems to obtain large dynamic range and high spatial resolution was demonstrated [23] in 2011. Hu et al. [24] proposed a unique photon-counting OTDR based on superconducting nanowire single-photon detectors (SNSPD), monitoring a $0.04 \mathrm{~cm}$ spatial resolution and a sensing length of $110 \mathrm{~km}$. However, the high sensitivity of the detector is realized via electrical amplification, which requires low temperature $(2 \mathrm{~K} \sim 4 \mathrm{~K})$ to suppress noise.

In this work, we theoretically and experimentally report a novel direct-detection BOTDR sensor system for distributed temperature measurement based on the combination of the intensity ratio of the Rayleigh signal and the anti-Stokes (RASR) method and single-photon detection technology, where the separation of the anti-Stokes component from the Rayleigh signal is achieved by a cascading FBG filter and then the ambient temperature information is calculated by the RASR. Meanwhile, a time-corrected singlephoton counting (TCSPC) method is adopted to generate the histograms of the time-of-flight delays between the incident of a probe light pulse and the capture of backscattered photons. The maximum dynamic range can reach $20 \mathrm{~dB}$ (corresponding to $120 \mathrm{~km}$ sensing range, considering that the attenuation ratio of the FUT is about $0.168 \mathrm{~dB} / \mathrm{km}$ in our experiment) without any optical amplification. Additionally, the experiment results also demonstrated that the proposed method is suitable to realize high spatial resolution and acceptable measurement error as well as large sensing length in a relatively simple scheme, and a spatial resolution about $1.2 \mathrm{~m}$ and a temperature measurement error about $1.24^{\circ} \mathrm{C}$ are obtained in $4.2 \mathrm{~km}$ sensing range.

\section{Theory}

According to the previous experimental results [18], the distributed direct-detection temperature sensing system is based on the fact that only the backscattered SpBS component is sensitive to the ambient temperature, while the backscattered RS component is insensitive to the surrounding environment temperature and generally acts as a reference. Thus, it is not necessary to obtain the absolute intensity ratio (Rayleigh/(Stokes + anti-Stokes)) to calculate the temperature information along the sensing FUT. In addition, it is not easy to obtain Stokes and anti-Stokes component with the proposed filter method at the same time. Therefore, the RASR method is presented to demodulate the ambient temperature information along the FUT, which is an excellent candidate for the absolute intensity ratio measurement. Clearly, compared with the measurement of SpBS signal in traditional method, the proposed method is more convenient and only needs a single monitor of the anti-Stokes signal. As a result, the absolute ambient temperature information relating to the relative change of the ratio of RASR can be given by the following equation:

$$
T=\frac{1}{C_{T}}\left(1-\frac{\operatorname{RASR}(T)}{\operatorname{RASR}\left(T_{\text {reference }}\right)}\right)+T_{\text {reference }}
$$

where $C_{T}$ represents the temperature sensitivity of the Brillouin-based distributed temperature sensor, $0.3 \% /{ }^{\circ} \mathrm{C}$ as common. It means that the absolute ambient temperature information relating to the relative change of the ratio of RASR meets the linear relationship with the coefficient of $0.3 \% /{ }^{\circ} \mathrm{C}$. $\operatorname{RASR}(T)$ and $\operatorname{RASR}\left(T_{\text {reference }}\right)$ are the intensity ratio of the RS and anti-Stokes signal of the unknown ambient temperature and the known ambient temperature, respectively. $T$ reference and $T$ represent the reference temperature value (i.e., the FUT at known ambient temperature) and the unknown temperature value, respectively. 
It is apparent that the proposed method can be used to monitor ambient temperature variation along the length of the FUT link. However, if the measured anti-Stokes power includes the residual RS component after cascading filter, a significant temperature error will occur when performing RASR measurement. The rejection ratio is defined as the ratio of the Brillouin anti-Stokes power $\left(P_{\text {anti-Stokes }}\right)$ to the residual RS power $\left(P_{\text {residual }}\right)$. It can be described as follows:

$$
\mathrm{RR}=\frac{P_{\text {anti-Stokes }}}{P_{\text {residual }}} \text {. }
$$

To demonstrate the difference between the actual temperature value and the nominal temperature value (i.e., temperature information measurement error) under different levels of Rayleigh noise, a simulation is implemented. In simulation, we choose the $\operatorname{RASR}\left(T_{\text {reference }}\right)$ at room temperature $\left(20^{\circ} \mathrm{C}\right)$ as a reference. Figure 1 highlights the results of the simulation under different $R R$ values. It can be seen from Figure 1 that the ambient temperature measurement error increases with the increase of the residual RS. When the rejection ratio exceeds $20 \mathrm{~dB}$, the impact of the residual RS power on temperature measurement error is negligible, and the measurement errors are $0.43^{\circ} \mathrm{C}$ and $0.14^{\circ} \mathrm{C}$ at $70^{\circ} \mathrm{C}$, respectively. It is worth noting that the $\mathrm{RR}$ reaching $25 \mathrm{~dB}$ is very difficult, which is limited according to the performance of the cascading FBG filter. Furthermore, not only will the power of the RS signal deteriorate the temperature measurement accuracy, but also the coherent noise of the backscattered RS from the narrow bandwidth laser source would lead to the temperature measurement error [25]. Therefore, the actual measurement inaccuracy will be larger than the theoretical result.

\section{Experimental Setup}

A schematic diagram of the experimental arrangement is presented in Figure 2. An ultranarrow linewidth $(\sim 10 \mathrm{kHz})$ distributed feedback (DFB) laser is used as the light source, when the SpBS signal is measured. Nominal power is about $15 \mathrm{~dB}$ m with a vacuum emission central wavelength of $1549.97 \mathrm{~nm}$. The output of the continuous wave laser source is shaped to probe pulse by a cascading electro-optic modulator (i.e., the combination of EOM1 and EOM2) with a $55 \mathrm{~dB}$ high extinction ratio (ER), which is driven by an arbitrary function generator (AFG). Two polarization controllers (PC1 and PC2) are employed just before the EOM1 and EOM2 module to realize the shaped output probe light pulse with good performance, respectively. Then, the modulated probe pulse is launched into a polarization scrambler (PS) to mitigate the polarization-reduced noise problem and directed into the other end of the sensing FUT (YOFC, HT1510-B) through a circulator (CIR). The backscattered sensing information is obtained in the same access. A cascading FBG filter, which consists of a common FBG1 filter ( $3 \mathrm{~dB}$ bandwidth of about $0.24 \mathrm{~nm}$ ) and a thermally adjustable narrowband FBG2 filter with $3 \mathrm{~dB}$ bandwidth of 40pm (AOS Gmbh, 18062083), in conjunction with the second optical switch (OS2) is employed to pick out the antiStokes component against the strong backscattered RS signal. Finally, the sensing signal of the backscattered SpBS is captured by an SPD (Qasky, WT-SPD300-ULN) and a TCSPC (PicoQuant, PicoHarp300) for follow-up data processing to retrieve the temperature information. When performing the so-called strong backscattered RS component measurement, an amplified spontaneous emission (ASE) light source with the maximum output power of $16.9 \mathrm{~dB} \mathrm{~m}$ is used instead of the DFB laser source to suppress the fluctuation of captured backscattered RS caused by coherent fading noise. The received wideband backscattered $\mathrm{RS}$ is coupled into the variable optical attenuator (VOA) via the second channel of the OS2 in order to avoid saturating the SPD. It should be noted that the captured sensing signal coupled into the VOA includes not only the backscattered RS signal but also the backscattered Stokes and anti-Stokes Brillouin signal. But the weight of SpBS compared with that of backscattered RS signal is negligible, so it has little effect on the following calculation of the intensity ratio of the Rayleigh signal and the anti-Stokes signal. At last, the measured backscattering Rayleigh photon counts $\left(N_{\text {Rayleigh }}\right)$ and Brillouin anti-Stokes photon counts ( $\left.N_{\text {anti-Stokes }}\right)$ histogram distributions are obtained on the TCSPC, respectively, and the ambient temperature trace can be plotted from $N_{\text {Rayleigh }} / N_{\text {anti-Stokes }}$.

\section{Experimental Results and Discussion}

In the experiment, to demonstrate the filtering performance of the cascading FBG filter, we first analyze the spectral characteristics of the thermally adjustable narrowband FBG2 filter and the linear relationship between the output filter wavelength and the input dialing value. By using the ASE light source and an optical spectrum analyzer (OSA: YOKOGAWA, AQ6370D), the corresponding experimental results are successfully obtained, which are separately plotted in Figures 3 and 4.

As displayed in Figure 3, the filtering performance of the thermally adjustable narrowband FBG2 filter is stable. The output filter wavelength is changed when the dialing value changed, and moves to the larger wavelength as the dialing value increases. Figure 4 highlights the output filter wavelength, plotted versus the input dialing value. Meanwhile, a least squares fitting of linear regression is used in order to demonstrate the linear relationship. It can be seen that the output filter wavelength increases with the input dialing value increasing, and the experimental results are in good agreement with the linear fitting result.

Subsequently, an experiment is carried out to determine the capability of the cascading FBG filter that a pure backscattered Brillouin anti-Stokes component can achieve in the sensing FUT. A DFB laser source is employed to introduce Rayleigh and spontaneous Brillouin signals. The monitored spectra with and without the cascading FBG filter are depicted in Figure 5 by means of an OSA. As expected, the Brillouin anti-Stokes signal is extracted from the backscattering RS light with RR no less than $21 \mathrm{~dB}$ when utilizing the proposed filter method. Therefore, the influence of RS noises on the anti-Stokes signal could be negligible. 


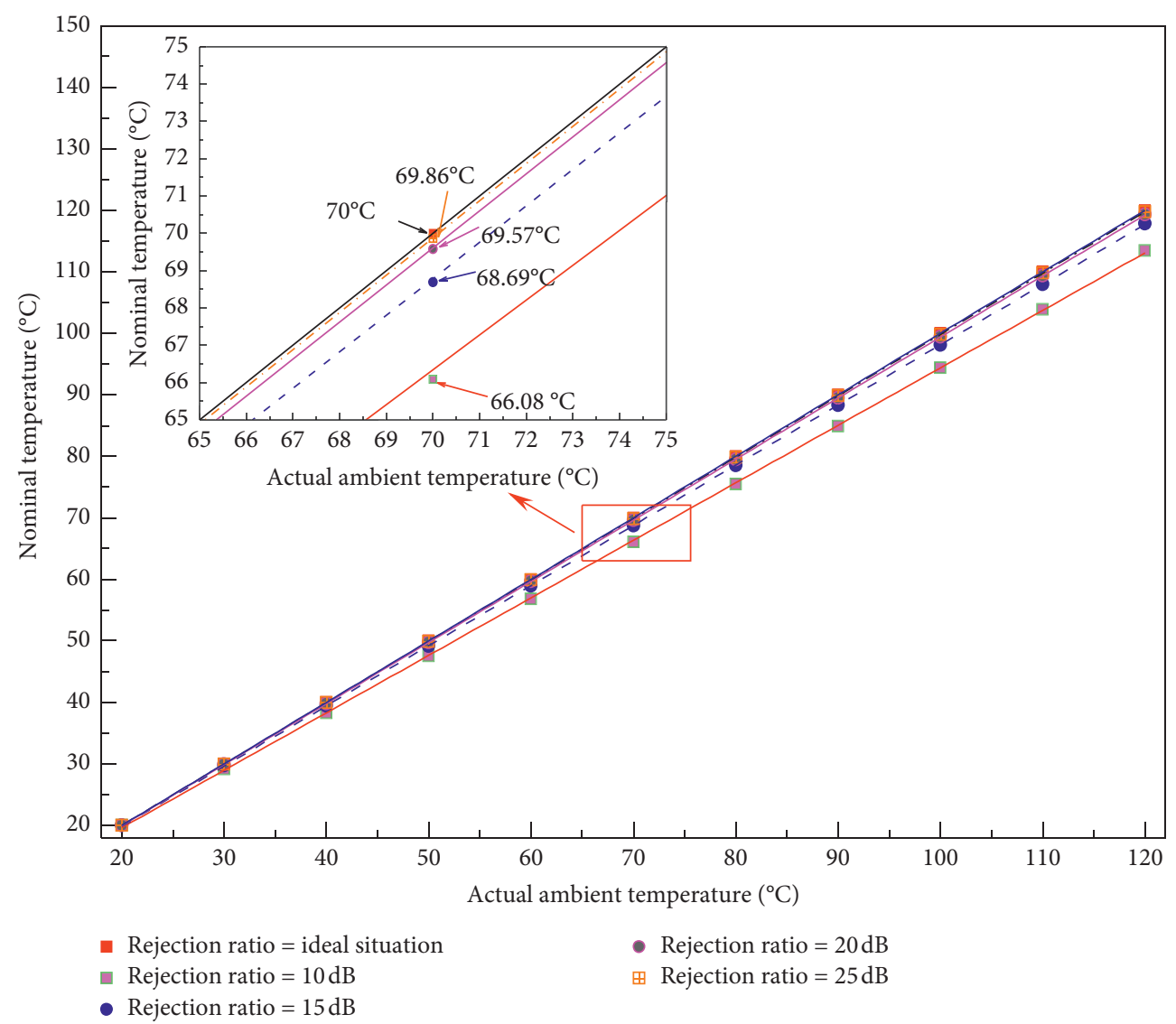

FIgURE 1: Simulation results of ambient temperature measurements under different rejection ratios.

In addition, to fully demonstrate the advantages of the presented BOTDR DTS sensor, temperature measurement is carried out by placing a segment of the FUT into a thermally insulated oven to simulate the change of environmental temperature. We carry out two types of experiment. The first type of experiment focuses on long-distance measuring of temperature information. The second one puts particular emphasis on the achievable spatial resolution. For the longdistance temperature measurement, a $1 \mathrm{~km}$ segment (the third section) of the $34 \mathrm{~km}$ standard SMF is put into a thermally insulated oven, which is located at the position between the second section (about $31 \mathrm{~km}$ ) and the fourth section (about $1 \mathrm{~km}$ ), and kept at a temperature of $50^{\circ} \mathrm{C}$. The continuous wave probe light is modulated to form a $1 \mu$ s pulse, corresponding to $100 \mathrm{~m}$ spatial resolution, and the repetition rate is $1 \mathrm{kHz}$, which depends on the length of the monitored fiber. In order to enhance the dynamic range, a $5 \mathrm{~ns}$ gate width and $5 \mu$ s dead time with detection efficiency of $10 \%$ are applied to the SPD. Meanwhile, the TCSPC records the histograms of the time-offlight delays between the incident probe light and the detection of anti-Stokes photons at the SPD with a $262 \mathrm{~ns}$ time bin width. The captured data are converted into standard photoncounting OTDR traces using the measured $N_{\text {Rayleigh }}$ and $N_{\text {anti-Stokes. }}$. Furthermore, what deserves special mention is that the measured $N_{\text {Rayleigh }}$ is not sensitive to the ambient temperature and can be monitored before the true sensing experiment. Therefore, in practical applications, only $N_{\text {anti-Stokes }}$ is needed to be measured.

Figure 6 illustrates the relationship between the backscattered signal power and the sensing range. The red solid line denotes the RS component and the blue solid line represents the SpBS signal. The linear fitting results of the experimental data show that the attenuation coefficient of the $34 \mathrm{~km}$ sensing optical fiber is $0.168 \mathrm{~dB} / \mathrm{km}$, which is in good agreement with the nominal loss value of $0.18 \mathrm{~dB} / \mathrm{km}$. It is evident that the backscattered power of the heated zone is distinguished. By calculating the noise value away from the trailing end of the FUT, the peak dynamic range of the proposed BOTDR DTS system with about $20 \mathrm{~dB}$ can be obtained, which corresponds to a temperature measuring distance of about $120 \mathrm{~km}$. According to the statistical distribution histograms of the measured RS and anti-Stokes photon, the calculated temperature information through RASR algorithm versus distance is plotted in Figure 7. It can therefore be observed that the temperature difference between the reference room temperature $\left(20^{\circ} \mathrm{C}\right)$ with the $\operatorname{RASR}\left(T_{\text {reference }}\right)$ of 30.45 and the true measured temperature with the RASR $(T)$ of 27.62 is $30.96^{\circ} \mathrm{C}$ using the proposed method. Obviously, the measured ambient temperature $\left(50.96^{\circ} \mathrm{C}\right)$ is in agreement with the real ambient temperature and presents a $0.96^{\circ} \mathrm{C}$ temperature error. It is noteworthy that the RASR value under such fluctuating background can 


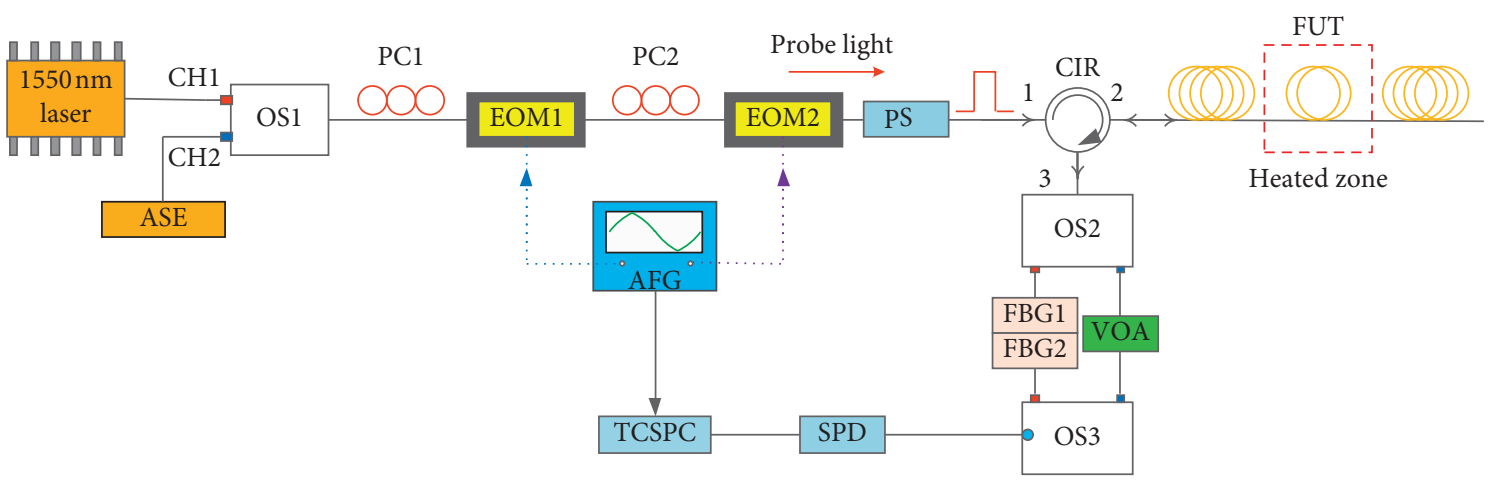

FIGURE 2: Experimental setup of distributed Brillouin temperature sensing system based on single-photon detector. EOM, electro-optic modulator; FUT, fiber under test; PC, polarization controller; AFG, arbitrary function generator; SPD, single-photon detector; FBG, fiber Bragg grating; VOA, variable optical attenuator; TCSPC, time-correlated single-photon counting; CIR, circulator; PS, polarization scrambler; OS, optical switch.

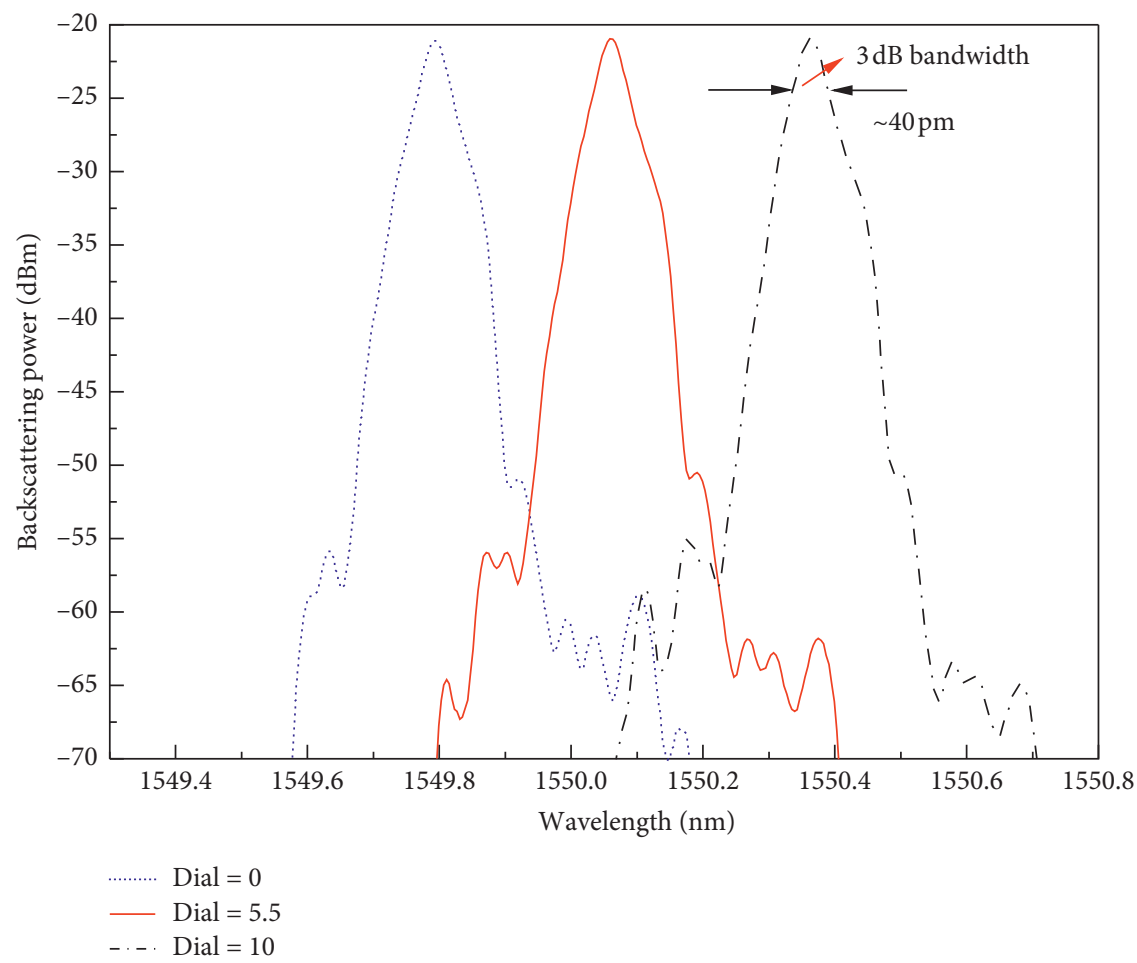

FIGURE 3: Observed spectral characteristics of the thermally adjustable narrowband FBG2 filter as the dialing value is 0 , 5.5, and 10, respectively.

be obtained along the FUT, given by averaging of the five continuous measurement results.

In the spatial resolution experiment, except for the time bin width of TCSPC (2 ns), other parameters of the SPD are consistent with the first type experiment. As the spectral linewidth of the probe pulse becomes comparable to the SpBS linewidth, one expects a reduction in Brillouin amplification factor. The spontaneous Brillouin linewidth is directly related to acoustic phonon lifetime (typically not larger than $10 \mathrm{~ns}$ ) in the FUT. Therefore, the optical probe pulse width controlled by the cascading EOM is $10 \mathrm{~ns}$, corresponding to $1 \mathrm{~m}$ spatial resolution, to make full use of the SpBS. The measured FUT is composed of five spools of SMF with the four equal lengths of about $1 \mathrm{~km}$ and a $200 \mathrm{~m}$. The second and fourth section of the FUTs to be measured are placed in a thermally insulated oven and maintained at a temperature of $55^{\circ} \mathrm{C}$ and a temperature of $50^{\circ} \mathrm{C}$, respectively. Figure 8 highlights the experimental results of the measured temperature value as a function of sensing length. We can find that the change in room sections $\left(20^{\circ} \mathrm{C}\right)$ and the heated sections of the FUTs are $36.24^{\circ} \mathrm{C}$ with a measured temperature error of $1.24^{\circ} \mathrm{C}$ and $31.41^{\circ} \mathrm{C}$ with a measured temperature error of $1.41^{\circ} \mathrm{C}$, respectively. Additionally, as depicted in Figure 9, a spatial resolution of $1.2 \mathrm{~m}$ is demonstrated along the 


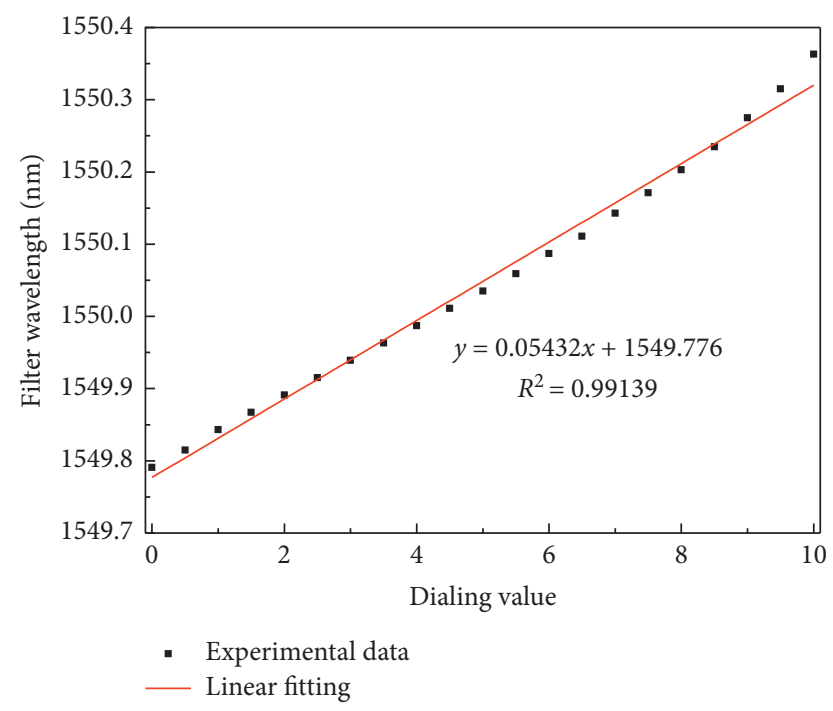

FIGURE 4: Measuring the output filter wavelength versus the input dialing value.

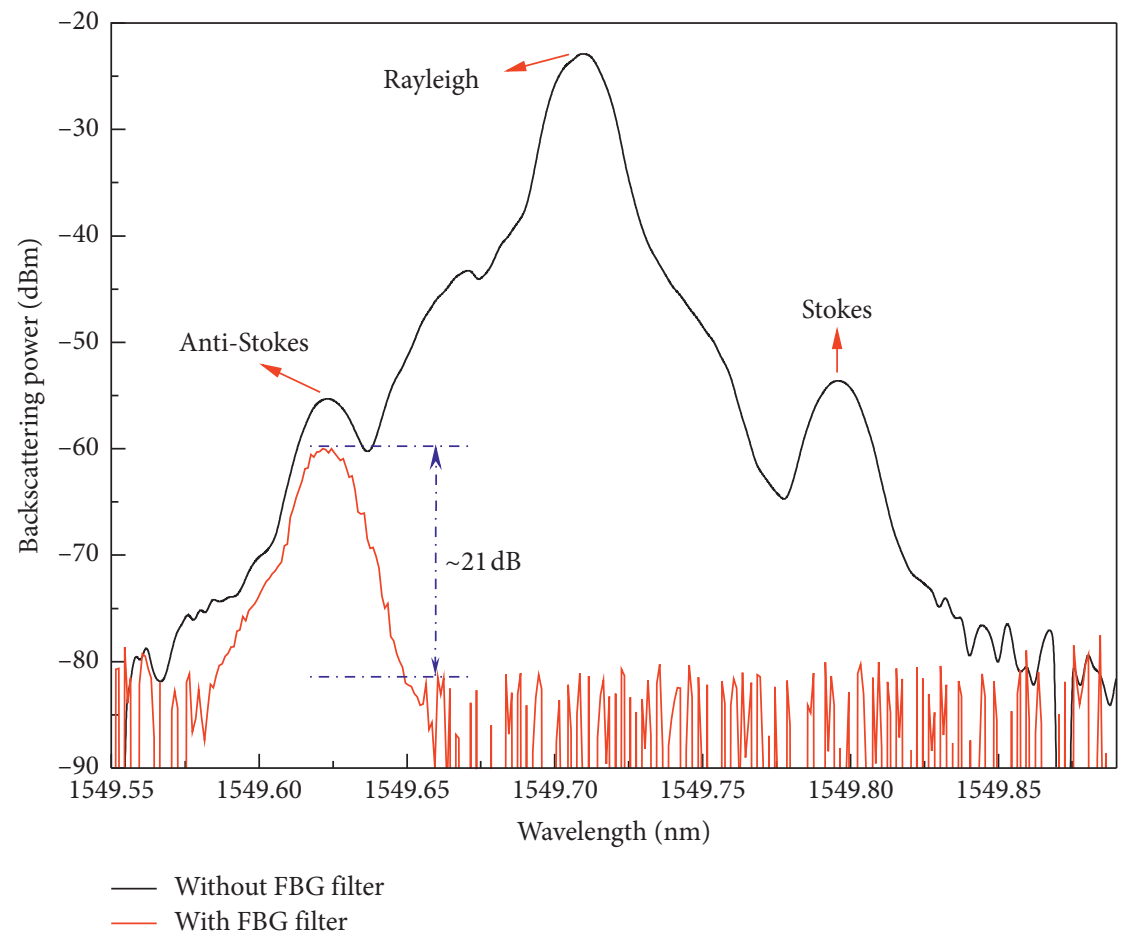

FIGURE 5: Measuring the backscattered spectra with and without the cascading FBG filter.

FUT of $4.2 \mathrm{~km}$, which is slightly larger than the theoretical spatial resolution of $1 \mathrm{~m}$ due to the fluctuation of the counts and the broadened pulse by the chromatic dispersion in the FUT. Certainly, the fluctuation of the counts could be efficiently improved by increasing the average times. 


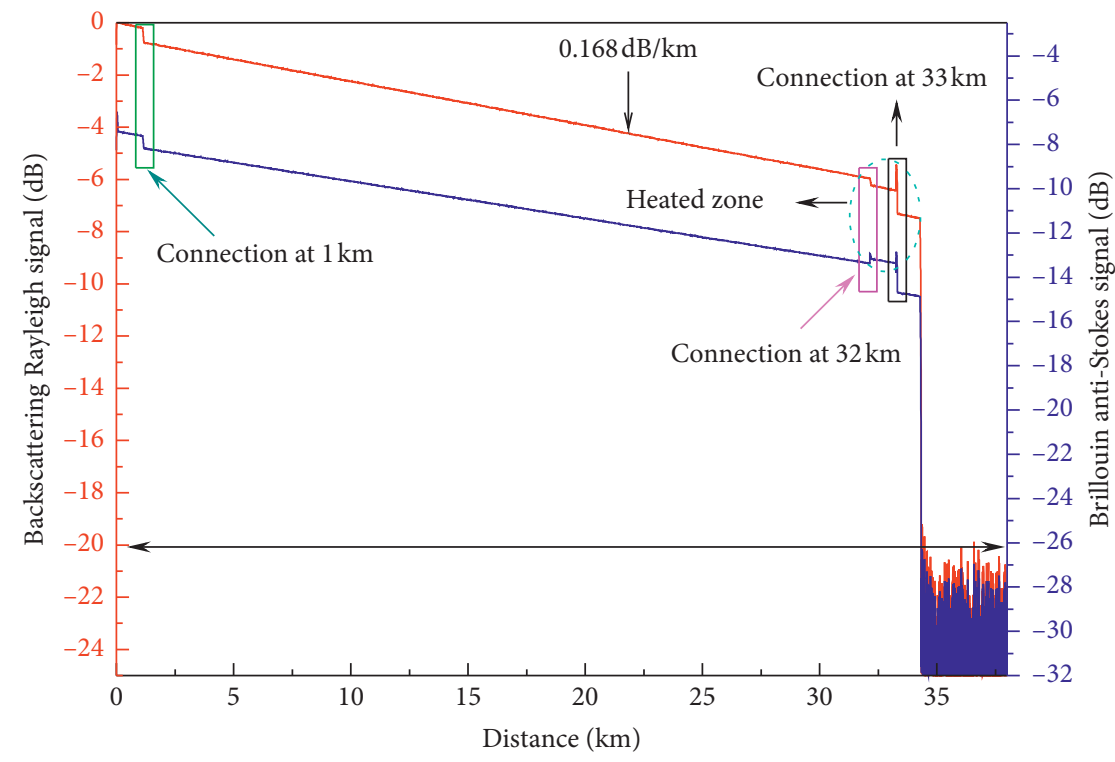

Figure 6: Measuring backscattered Rayleigh and anti-Stokes Brillouin signal traces under $1 \mu$ s pulse.

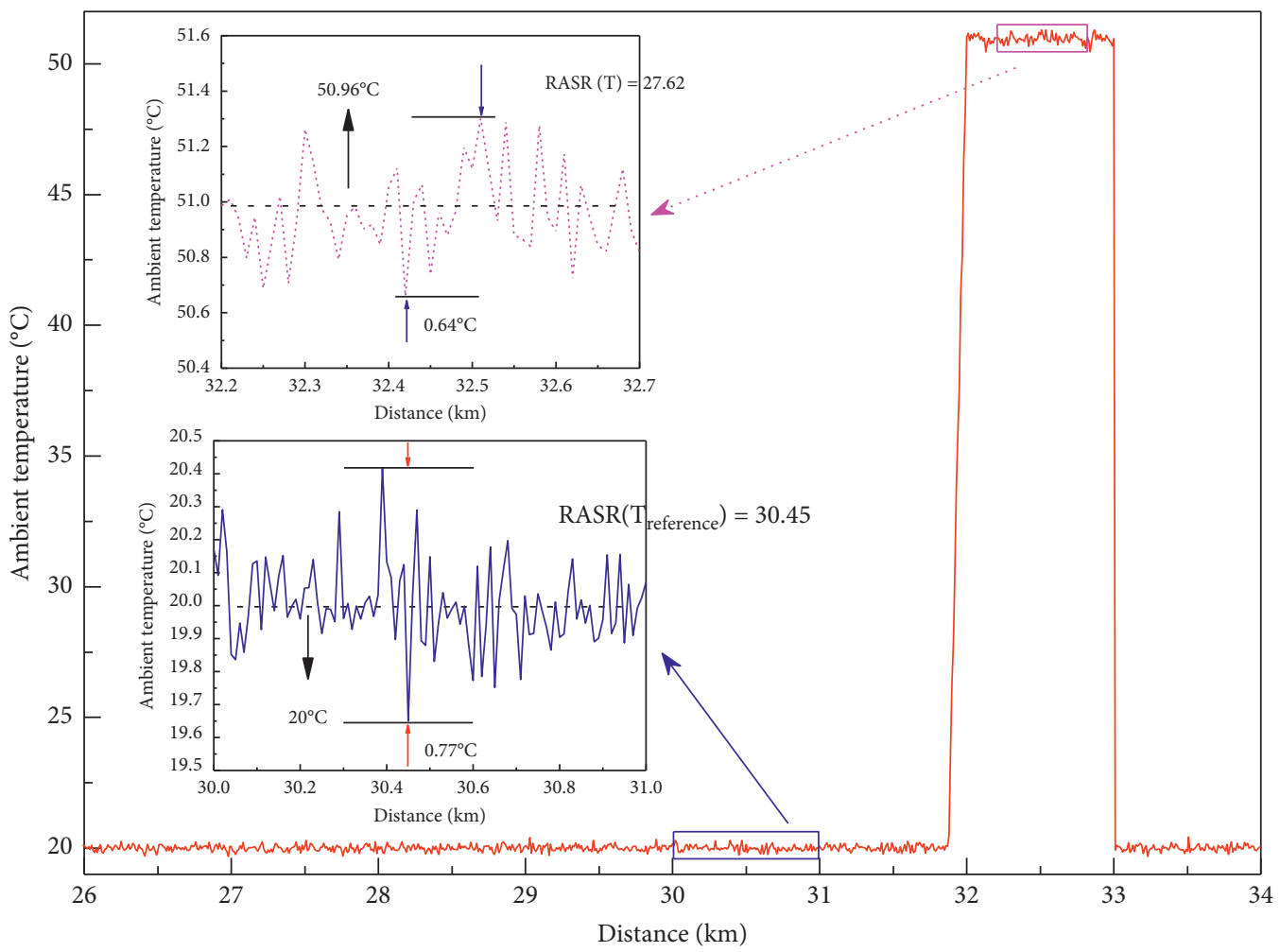

Figure 7: Measured temperature profiles along the sensing fiber. 


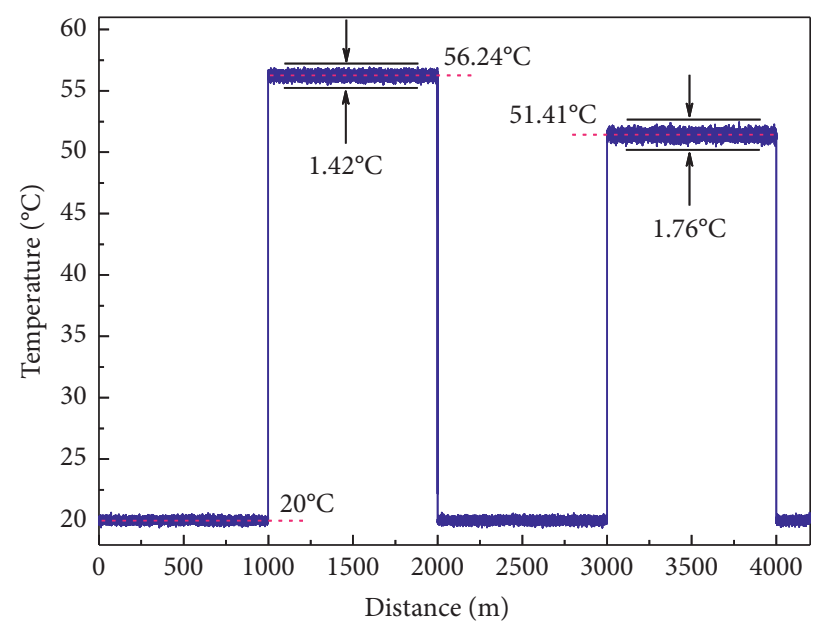

Figure 8: Measured temperature profiles along the FUT, with standard deviation labeled in each heated segment.

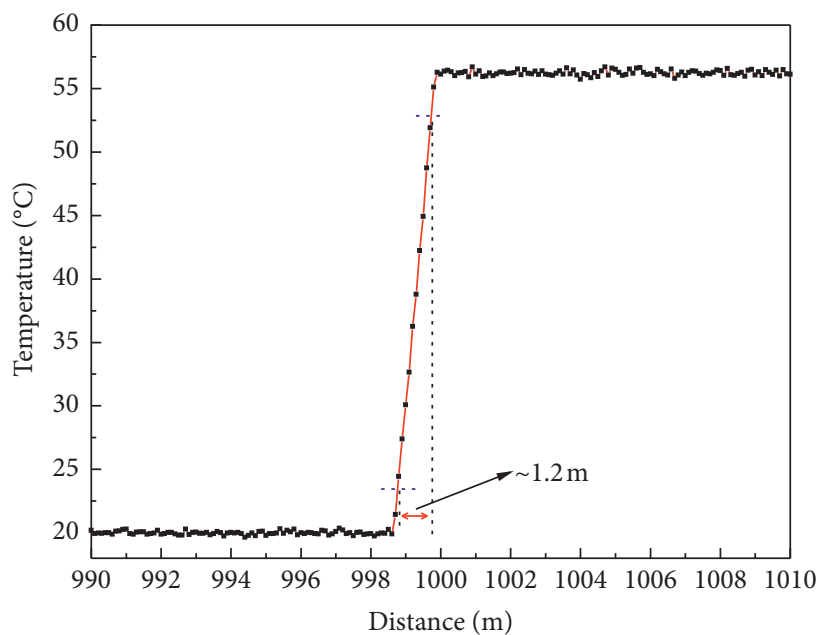

Figure 9: Spatial resolution measurement of the Brillouin sensing system.

\section{Conclusions}

In this work, we demonstrate a direct-detection Brillouin-based distributed temperature system based on a photon-counting detector. The capability of measurement temperature with large dynamic range and high spatial resolution is experimentally investigated in detail. A dynamic range of $20 \mathrm{~dB}$, corresponding to the sensing length of $120 \mathrm{~km}$, is successfully presented with a measured temperature error of $0.96^{\circ} \mathrm{C}$. Meanwhile, the proposed photon-counting sensing system also exhibits a spatial resolution of $1.2 \mathrm{~m}$ and a measured temperature error of $1.24^{\circ} \mathrm{C}$ in $4.2 \mathrm{~km}$ sensing range. The performance of Brillouin-based distributed temperature sensor may be further improved by increasing the incident probe light power without exciting other nonlinear effects and shortening the injection pulse width.

\section{Data Availability}

The data used to support the findings of this study have not been made available because the group is a confidential unit and the experimental data are mainly used for the development of related instruments.

\section{Conflicts of Interest}

The authors declare that they have no conflicts of interest.

\section{Acknowledgments}

The authors gratefully acknowledge the financial support provided by the Science and Technology on Electronic Test and Measurement Laboratory Foundation (Grants 41Q1313-5 and KDW03012003), the Taishan Series Talent Project (Grant 2017TSCYCX-05), and the National Natural Science Foundation of China (Grant 61901426).

\section{References}

[1] E. Garmire, "Stimulated Brillouin review: invented 50 years ago and applied today," International Journal of Optics, vol. 2018, Article ID 2459501, 17 pages, 2018. 
[2] Z. X. Bai, Y. L. Wang, Z. H. Liu et al., "Demonstration of an ultraviolet stimulated Brillouin scattering pulse compressed hundred picosecond laser in $\mathrm{LiB}_{3} \mathrm{O}_{5}$ crystals," Journal of Optics, vol. 19, no. 8, Article ID 85502, 2017.

[3] Z. X. Bai, R. J. Williams, O. Kitzler et al., "Diamond Brillouin laser in the visible," APL Photonics, vol. 5, no. 3, Article ID 31301, 2020.

[4] Z. Bai, H. Yuan, Z. Liu et al., "Stimulated Brillouin scattering materials, experimental design and applications: a review," Optical Materials, vol. 75, pp. 626-645, 2018.

[5] Z. Bai, Y. Wang, Z. Yuan et al., "High compact, high quality single longitudinal mode hundred picoseconds laser based on stimulated Brillouin scattering pulse compression," Applied Sciences, vol. 6, no. 1, p. 29, 2016.

[6] L. Sheng, D. Ba, and Z. Lu, "Imaging enhancement based on stimulated Brillouin amplification in optical fiber," Optics Express, vol. 27, no. 8, pp. 10974-10980, 2019.

[7] L. Sheng, D. Ba, and Z. Lu, "Low-noise and high-gain of stimulated Brillouin amplification via orbital angular momentum mode division filtering," Applied Optics, vol. 58, no. 1, pp. 147-151, 2019.

[8] L. W. Sheng, D. X. Ba, and Z. W. Lu, "Weak laser pulse signal amplification based on a fiber Brillouin amplifier," Chinese Optics Letters, vol. 16, no. 11, Article ID 111901, 2018.

[9] X. Bao and L. Chen, "Recent progress in distributed fiber optic sensors," Sensors, vol. 12, no. 7, pp. 8601-8639, 2012.

[10] X. Bao and L. Chen, "Recent progress in Brillouin scattering based fiber sensors," Sensors, vol. 11, no. 4, pp. 4152-4187, 2011.

[11] L. Sheng, L. Li, L. Liu, L. Hu, M. Yuan, and J. Yan, "Study on the simultaneous distributed measurement of temperature and strain based on Brillouin scattering in dispersion-shifted fiber," OSA Continuum, vol. 3, no. 8, pp. 2078-2085, 2020.

[12] L. W. Sheng, L. G. Li, L. J. Hu et al., "Distributed fiberoptic sensor for simultaneous temperature and strain monitoring based on Brillouin scattering effect in polyimide-coated fibers," International Journal of Optics, vol. 2020, Article ID 8810986, 5 pages, 2020.

[13] X. Bao, D. J. Webb, and D. A. Jackson, "32-km distributed temperature sensor based on Brillouin loss in an optical fiber," Optics Letters, vol. 18, no. 18, pp. 1561-1563, 1993.

[14] D. W. Zhou, Y. K. Dong, B. Z. Wang et al., "Single-shot BOTDA based on an optical chirp chain probe wave for distributed ultrafast measurement," Light: Science \& Applications, vol. 7, no. 1, p. 32, 2018.

[15] B. Wang, B. Fan, D. Zhou et al., "High-performance optical chirp chain BOTDA by using a pattern recognition algorithm and the differential pulse-width pair technique," Photonics Research, vol. 7, no. 6, pp. 652-658, 2019.

[16] A. Coscetta, A. Minardo, L. Olivares et al., "Wind turbine blade monitoring with Brillouin-based fiber-optic sensors," Journal of Sensors, vol. 2017, Article ID 9175342, 5 pages, 2017.

[17] S. M. Maughan, H. H. Kee, and T. P. Newson, " $57-\mathrm{km}$ single-ended spontaneous Brillouin-based distributed fiber temperature sensor using microwave coherent detection," Optics Letters, vol. 26, no. 6, pp. 331-333, 2001.

[18] J. Schroeder, R. Mohr, P. B. Macedo, and C. J. Montrose, "Rayleigh and Brillouin scattering in $\mathrm{K} 2 \mathrm{O}-\mathrm{SiO} 2$ glasses," Journal of the American Ceramic Society, vol. 56, no. 10, pp. 510-514, 1973.

[19] P. C. Wait and A. H. Hartog, "Spontaneous Brillouin-based distributed temperature sensor utilizing a fiber Bragg grating notch filter for the separation of the Brillouin signal," IEEE Photonics Technology Letters, vol. 13, no. 5, pp. 508-510, 2001.
[20] P. C. Wait and T. P. Newson, "Landau Placzek ratio applied to distributed fibre sensing," Optics Communications, vol. 122, no. 4-6, pp. 141-146, 1996.

[21] H. H. Kee, G. P. Lees, and T. P. Newson, “All-fiber system for simultaneous interrogation of distributed strain and temperature sensing by spontaneous Brillouin scattering," Optics Letters, vol. 25, no. 10, pp. 695-697, 2000.

[22] K. De Souza and T. P. Newson, "Brillouin-based fiber-optic distributed temperature sensor with optical preamplification," Optics Letters, vol. 25, no. 18, pp. 1331-1333, 2000.

[23] M. G. Tanner, S. D. Dyer, B. Baek et al., "High-resolution single-mode fiber-optic distributed Raman sensor for absolute temperature measurement using superconducting nanowire single-photon detectors," Applied Physics Letters, vol. 99, no. 20, Article ID 201110, 2011.

[24] J. Hu, Q. Zhao, X. Zhang et al., "Photon-counting optical time-domain reflectometry using a superconducting nanowire single-photon detector," Journal of Lightwave Technology, vol. 30, no. 16, pp. 2583-2588, 2012.

[25] K. D. Souza, "Significance of coherent Rayleigh noise in fibreoptic distributed temperature sensing based on spontaneous Brillouin scattering," Measurement Science and Technology, vol. 17 , no. 5 , p. $1065,2006$. 